EPJ Web of Conferences 114, 02144 (2016)

DOI: $10.1051 /$ epjconf/201611402144

C Owned by the authors, published by EDP Sciences, 2016

\title{
Distribution of velocity and turbulent characteristics in coarse-sediment laden flows above erodible plane bed in open channel
}

\author{
Štěpán Zrostlík ${ }^{1, a}$, Vojtěch Bareš ${ }^{1}$, Jan Krupička ${ }^{1}$, Tomáš Picek ${ }^{1}$, and Václav Matoušek ${ }^{1}$ \\ ${ }^{1}$ Department of Hydraulics and Hydrology, Czech Technical University in Prague, 16629 Prague 6
}

\begin{abstract}
Extreme conditions as flash floods in steep mobile-bed streams induce flows which carry a huge amount of sediment. Typically, the flow with the intense transport of sediment is stratified with a layered structure above an erodible plane bed. The paper deals with local flow characteristics in the layered structure of the two-phase flow composed of water and sediment. We present experimental data and results for two different fractions of plastic particles obtained using two acoustic Doppler methods and a Prandtl probe. The results contain vertical profiles of longitudinal velocity component and local turbulent characteristics of the flow as the Reynolds stress and turbulent intensity. The effect of the layered character of the flow on the flow characteristics is discussed. Special attention is paid to the distribution of Reynolds stress in the sediment-rich transport layer above the bed and to the evaluation of ability of carrier turbulent eddies to support sediment grains in and above the transport layer.
\end{abstract}

\section{Introduction}

The two-phase flow of coarse-sediment mixture occurs in mobile-bed open channels at extreme conditions as flash floods in mountain streams. A huge amount of transported material changes properties of the flowing medium and this needs to be considered while computing the flow rate and resistance of the flow [1] as well as [2]. The coarse-sediment flow occurs in the regime with bed forms or with no bed forms. In the bed-form free regime called the upper plane bed (UPB) regime, the flow resistance is significantly affected by the transported material. Flow in the UPB regime can be considered as a layered flow with three zones. The uppermost layer contains clear water and occasionally saltating particles. The transport layer is situated between the uppermost layer and the layer with deposit material. In the transport layer, a vast majority of the total load is transported. A UPB-based flow is described in details in [3] as well as [4] and its structure is shown in Figure 1. The logarithmic shape of a velocity profile is expected in the water layer and the linear shape is expected in the transport layer. Figure 1 shows also the expected linear concentration profile across the transport layer. This assumption are based on previous experimental observations in the UPB regime [5] as well as [6].

Distributions of local velocity and turbulent characteristics of flow are inevitable for better understanding of mechanisms governing flow structure and resistance. Measuring the distributions using classical optical methods as LDA or PIV is impossible because of high concentration of particles which does not allow to evaluate the point velocity in the center of a channel. Capart and Fraccarollo [6] used the modified optical method PTV (Particle Tracking Velocity) for measuring velocity profiles of transported particles near the side wall of the rectangular channel. Other authors used Prandtl probe for measuring the local velocity [7] and considered the measured velocities as velocities of water. Ultrasonic probes are utilized frequently. Rajesh et al. [8] and Song et al. [9] used Acoustic Doppler Velocimeter for measuring turbulence statistics above the erodible bed at relatively low transport concentration of sediment. Revil-Baudard et al. [10] measured velocity and concentration profiles with ACVP (Acoustic Concentration and Velocity Profiler) in mixture of bakelite grains and water. Bakhtyar et al. [11] used UVP (Ultrasonic Velocity Profiling) for measuring velocity characteristics above the sand bed under the condition of oscillatory sheet flow.

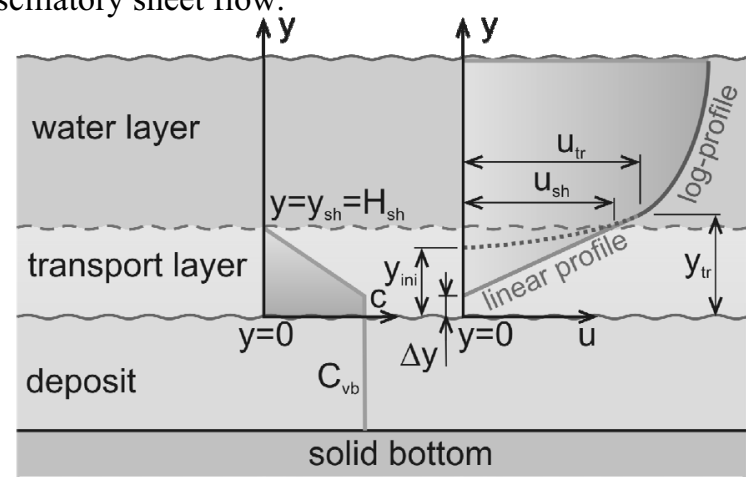

Figure 1. Scheme of flow in UPB regime with linear-log velocity distribution and linear concentration distribution

\footnotetext{
a Corresponding author: stepan.zrostlik@fsv.cvut.cz
} 
The work presented here is a continuation of our previous investigations on velocity distribution in stratified flows with both intense and weak transport of sediment. In this paper, we exploit our new experimental data to evaluate velocity profiles and focus on an evaluation of turbulent characteristics in the transport layer.

\section{Methods}

The experiments were carried out in the Water Engineering Laboratory of the Czech Technical University (CTU) in Prague. The experimental tilting flume and measured variables have been described elsewhere $[3,4]$.

\subsection{Measuring instruments}

We used three different measurement techniques to measure a vertical velocity profile of the longitudinal component of the velocity in the axis of the flume. The first technique is Prandtl probe (PT). It is a robust standard method and we used it primarily to validate velocity profiles obtained from the other methods. The second method used a UVP probe directed opposite to the flow. A disadvantage of the method in this particular application is that particles are in sporadic contact with the measuring membrane of the probe. Compared to the other two methods, the down-looking ADVP (Acoustic Doppler Velocity Profiler) is non-invasive while measuring within the transport layer. The probe senses the instantaneous 3-D velocity information along $32 \mathrm{~mm}$ long measuring section below the probe. The measuring instruments are described in more details and an estimation of their measuring errors is given in [3]. Processing of measured data is described in [3] as well as [4].

\subsection{Materials}

Experiments were carried out with two different fractions of plastic particles. Particles of both fractions are of a shape of a cylinder with one concave base and the other convex base. The diameter of the cylinders is $4.7 \mathrm{~mm}$ and the height for the coarser fraction is $4.9 \mathrm{~mm}$ (TLT50), the height for the smaller fraction is $2.2 \mathrm{~mm}$ (TLT25). Measured terminal settling velocity $v_{\mathrm{t}}$ of particles and diameters $\mathrm{d}_{50}$ of equivalent spherical particles of the same volume are presented in Table 1. Both particles have a similar density $\left(\rho_{\mathrm{s}}\right)$, they are made of PVC (TIULIT). Particles showed no signs of mechanical damage during the experiments. Overall, 44 runs were measured with TLT25 and 55 runs with TLT50.

Table 1. Properties of particles

\begin{tabular}{|c|c|c|}
\hline Properties & TLT25 & TLT50 \\
\hline$d_{50}(\mathrm{~mm})$ & 4.23 & 5.41 \\
\hline$v_{\mathrm{t}}\left(\mathrm{m} \cdot \mathrm{s}^{-1}\right)$ & 0.106 & 0.149 \\
\hline$\rho_{\mathrm{s}}\left(\mathrm{kg} \cdot \mathrm{m}^{-3}\right)$ & 1381 & 1307 \\
\hline
\end{tabular}

\subsection{Velocity profile}

A velocity profile of flow of water above the hydraulically rough boundary can be described using the logarithmic law of the wall [3],

$$
\frac{u}{u_{b}^{*}}=2.5 \ln \left(B \frac{y-y_{i n i}}{k_{s}}\right)
$$

where $u$ is longitudinal velocity at vertical position $y, u_{b}$ * is the bottom shear velocity, $y_{i n i}$ is position of origin of logarithmic profile, $k_{s}$ is the boundary roughness and $B$ is an integration constant for open channel flow $(B=30)$. The velocity distribution is linear in the transport layer and it can be described by following equation [3],

$$
\frac{u}{u_{t r}}=\left(\frac{y-\Delta y}{y_{t r}-\Delta y}\right)^{n}
$$

where $y_{t r}$ is vertical position, at which a profile smoothly transforms its shape from linear to logarithmic, $u_{t r}$ is velocity at position $y_{t r}, \Delta y$ is position of zero velocity and $n$ is an exponent $(\mathrm{n}=1)$.

Taking the above assumptions into account, we carried out an optimization procedure on measured velocity profiles resulting in values of five parameters $\left(u_{b}{ }^{*}, y_{i n i}, k_{s}\right.$, $\left.y_{t r}, \Delta y\right)$.

\subsection{Turbulent characteristics}

Knowledge of turbulent characteristics is vital for better understanding of particle movements in sediment-laden flows. By definition, the instantaneous velocity can be described as the time average velocity $\bar{u}$ plus the fluctuation component $u_{\mathrm{i}}^{\prime}$. For the longitudinal component of local velocity one can write,

$$
u_{i}=\bar{u}+u_{i}^{\prime}
$$

The same holds for the velocity in vertical direction, $w$. We consider the intensity of turbulence in the longitudinal direction as $\sqrt{\overline{u_{i}^{\prime 2}}} / u_{*}$. The local Reynolds stress in turbulent flow of water is defined as

$$
\tau_{x, \mathrm{z}}=-\rho_{w} \overline{u^{\prime} w^{\prime}}
$$

\section{Evaluation of velocity characteristics}

\subsection{Velocity distribution}

The velocity profiles, obtained using the different measuring methods, correspond with each other quite well (Figures 2 and 3). The lin-log shape of a velocity profile describes the measured vertical distribution of velocity very well, which is in an agreement with the earlier measurements with other sediment materials under the same condition [4]. Based on experimental data processed using the optimization procedure containing Equations (1) and (2), it is confirmed that the position of the transition in the trend of the velocity profile approximately corresponds with the position of the 
interface between the transport layer and the water layer. The slope of the linear profile remains approximately constant at very high shear stresses. The position of the virtual origin of the logarithmic part of a velocity profile is at of about 65 per cent of the height of the transport layer.

The upper plots of Figures 2 and 3 show examples of measured and processed velocity profiles at relatively low high stress, the lower plots show the profile at high shear stress. Experimental data from all three methods are plotted. The UVP data are from the distance 20, 30, 40 $\mathrm{mm}$ upstream the probe (UVP20, UVP30 and UVP40, respectively) and data from ADVP are from different installed vertical positions of the probe (ADVP1, ADVP2 etc.).
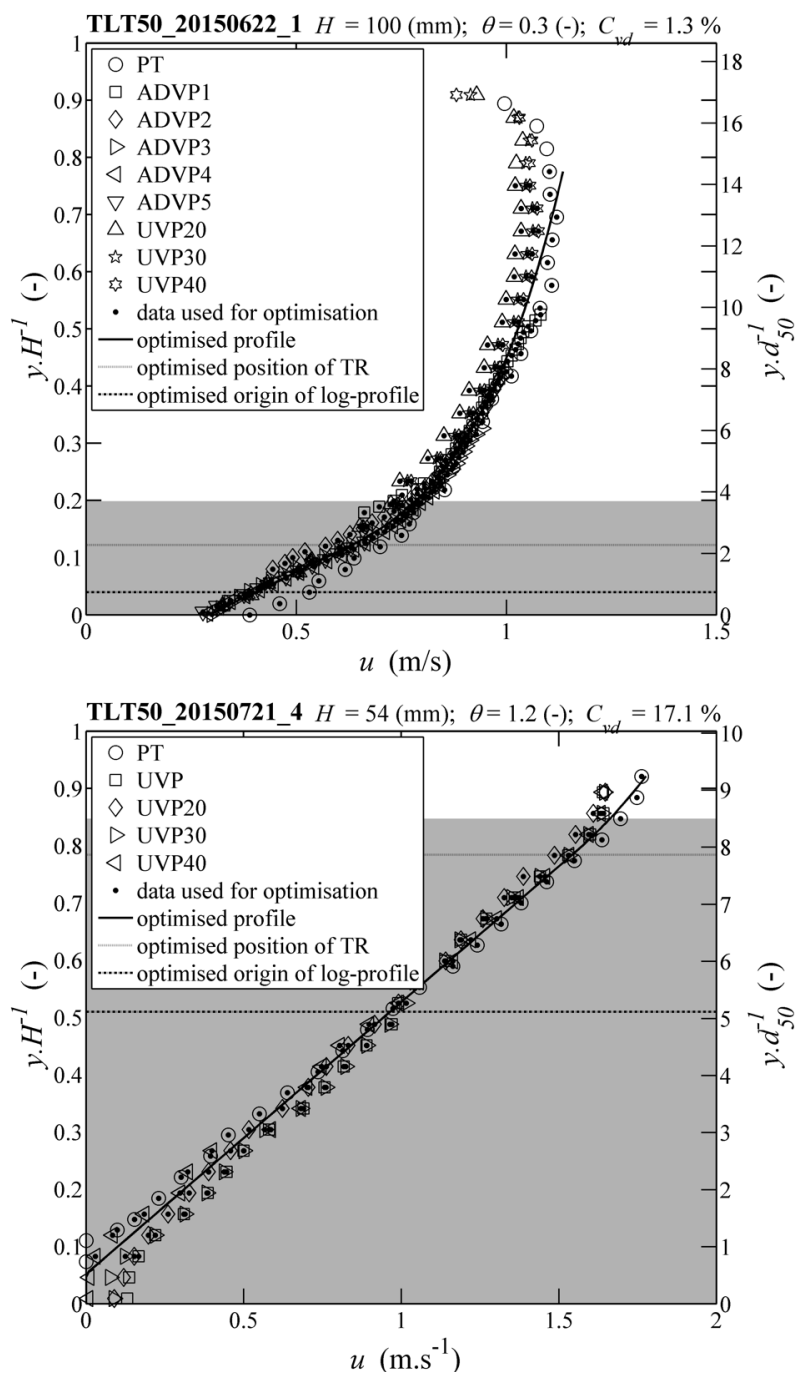

Figure 2. Measured and processed velocity profiles for TLT50. Legend: $C_{\mathrm{vd}}-$ delivered concentration of sediment, $\theta$ - Shields parameter, $H$ - depth of flow
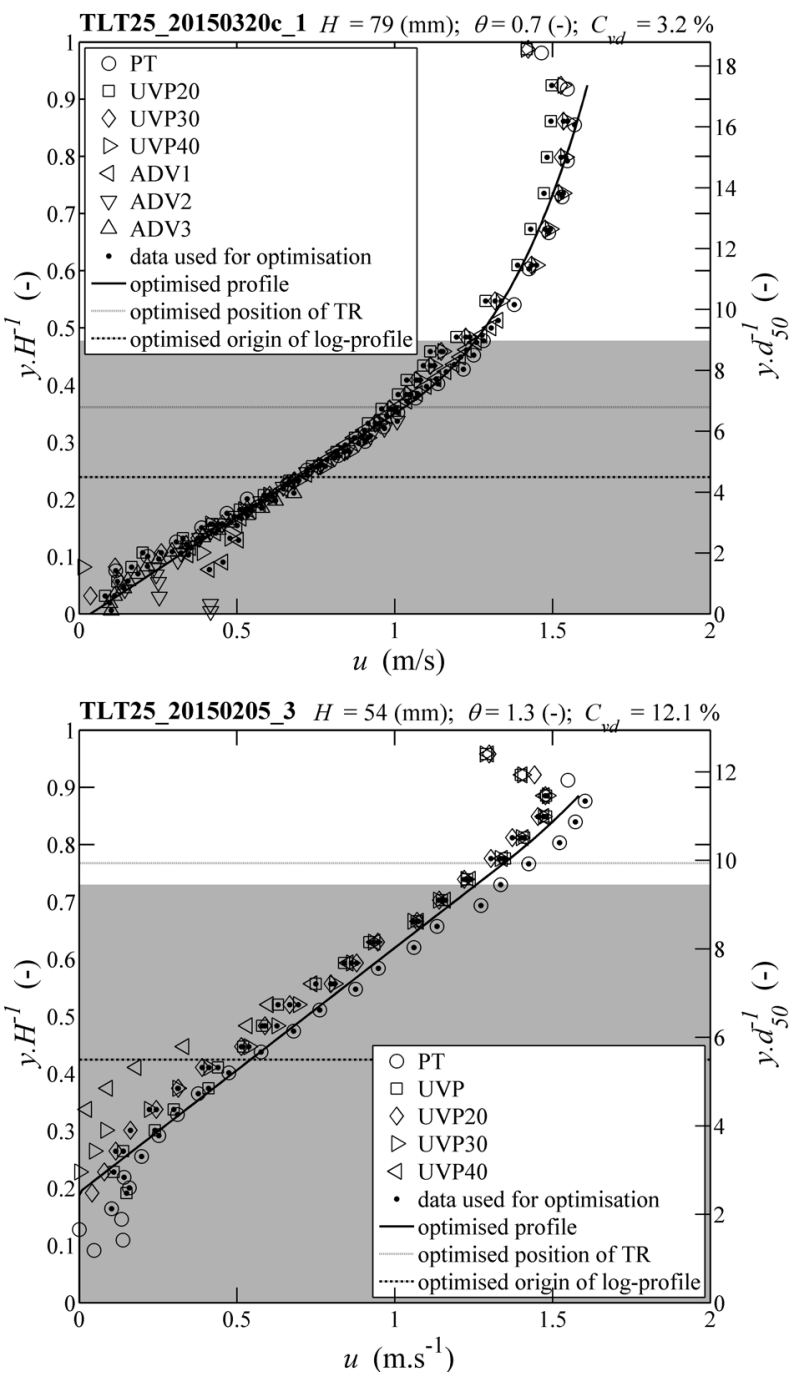

Figure 3. Measured and processed velocity profiles for TLT25. Legend: $C_{\mathrm{vd}}-$ delivered concentration of sediment, $\theta$ - Shields parameter, $H$ - depth of flow

We have employed two methods to evaluate the shear velocity $\left(u_{*}\right)$. The first approach estimates the shear velocity from the integral experimental data,

$$
u_{*_{\text {int }}}=\sqrt{g \cdot R_{b} \cdot \sin \beta}
$$

where $g$ is the gravitational acceleration, $\beta$ is the angle of the longitudinal slope of uniform flow and $R_{b}$ is the hydraulic radius associated with bed. The second method gives the shear velocity, $u_{*}$ opt, as a result of the optimization procedure to fit the theoretical velocity profile (Eq. 1) with the measured data in the water layer (i.e. in the theoretical inner region of the turbulent boundary layer). Figure 4 compares results of the two methods. The shear velocity from the velocity profile tends to be bigger than the shear velocity from the integral data and the difference increases with the increasing value of the shear velocity. It must be mentioned that there are some important differences which cause that the two velocities cannot be considered the same parameters. The first difference is caused by the spatial distribution of shear velocity along the width of 
the channel. The $u_{*}$ int data represent a width-averaged value of the shear velocity, whereas $u_{* \text { opt }}$ is evaluated for the plane of the vertical velocity profile and must be considered a local value. The second difference is caused by the effect of the vertical position. The $\mathrm{u}_{*} \mathrm{opt}_{\mathrm{t}}$ is optimized for the position of the origin of a logarithmic profile while $u *_{\text {in }}$ is determined for the top of the bed, i.e. the bottom of the transport layer.

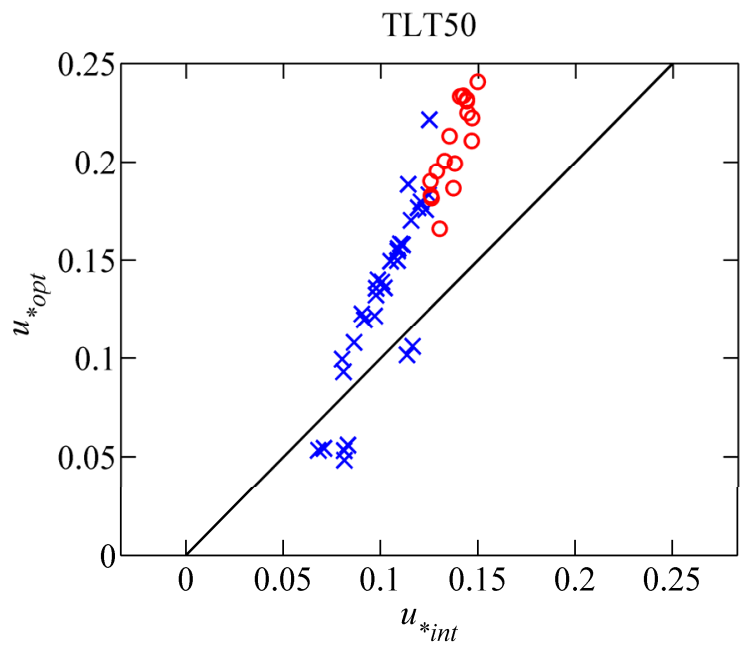

Figure 4. Comparison of shear velocity from integral data $\left(u_{* \text { int }}\right)$ and shear velocity from velocity profile $\left(u_{* \mathrm{opt}}\right)$. Legend: $\mathrm{x}$ - data for Shields number lower than 1,0 - data for Shields number higher than 1 , solid line - line of agreement

\subsection{Turbulent characteristics}

The turbulent characteristics were evaluated for all experimental data sets (corresponding with measured profiles) obtained using ADVP: 14 sets for TLT25 and 21 sets for TLT50. For longitudinal turbulent intensities, UVP measurements were used as well. Measurements from Prandtl probe were not considered for an analysis of turbulent characteristics.

\subsubsection{Longitudinal turbulent intensity}

Figure 5 shows evaluated longitudinal intensities of turbulence (the bed shear velocity from integral data is used to normalize the velocity fluctuation). The intensities obtained from the different measuring methods may differ in values, but exhibit the same trend. The intensity of turbulence increases approximately linearly from the water surface (the zero-value at the water surface) to the top of the transport layer. In the transport layer, the turbulence intensity starts to decrease with the increasing depth below the top of the layer. Apparently, damping of intensities is due primarily to the presence of particles and due to the increase of particle concentration with the depth within the transport layer. The presence of particles and their mutual collisions attenuate the longitudinal fluctuation associated with flow turbulence.
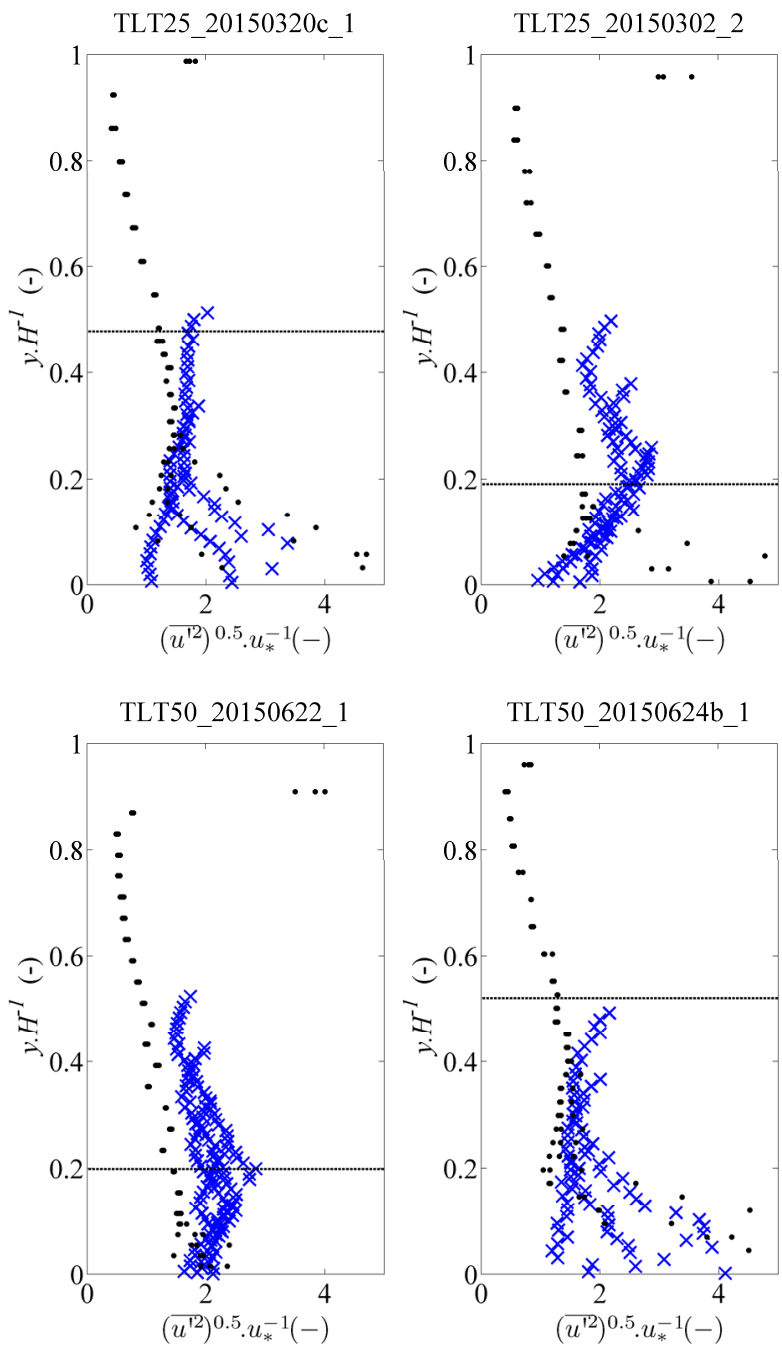

Figure 5. Distribution of longitudinal turbulent intensity (fluctuation normalized by bed shear velocity from integral data). Upper panels - TLT25, lower panels - TLT50. Legend: dots - UVP, $\mathrm{x}$ - ADVP, line - visually observed top of transport layer.

\subsubsection{Vertical turbulent intensity}

Instantaneous vertical velocity components were obtained by the ADVP probe only. Vertical pulsations were evaluated in the same way as the longitudinal ones. In Figure 6 , the hindered settling velocity $\left(v_{\text {th }}\right)$ is used to normalise the square root of the mean vertical fluctuation velocity. In the transport layer, the hindered settling velocity was determined from the Richardson-Zaki equation,

$$
v_{t h}=v_{t} \cdot\left(1-c_{v}\right)^{m}
$$

where $c_{v}$ is the local volumetric concentration of particles at given vertical position. The exponent $m$ was computed from the Wallis equation,

$$
m=\frac{4.7 \cdot\left(1+0.15 \cdot \operatorname{Re}_{p}^{0.687}\right)}{1+0.253 \cdot \operatorname{Re}_{p}^{0.687}}
$$


where $R e_{\mathrm{p}}$ is the particle Reynolds number. Figure 6 shows that the time-averaged vertical velocity fluctuation is considerably smaller than the terminal settling velocity of particles at the top of the transport layer. This suggests that support of particles comes from interparticle collisions rather than turbulent vortices at the top of the transport layer. The observed increase in values of the velocity ratio within the transport layer is due to the decreasing local hindered settling velocity of particles.
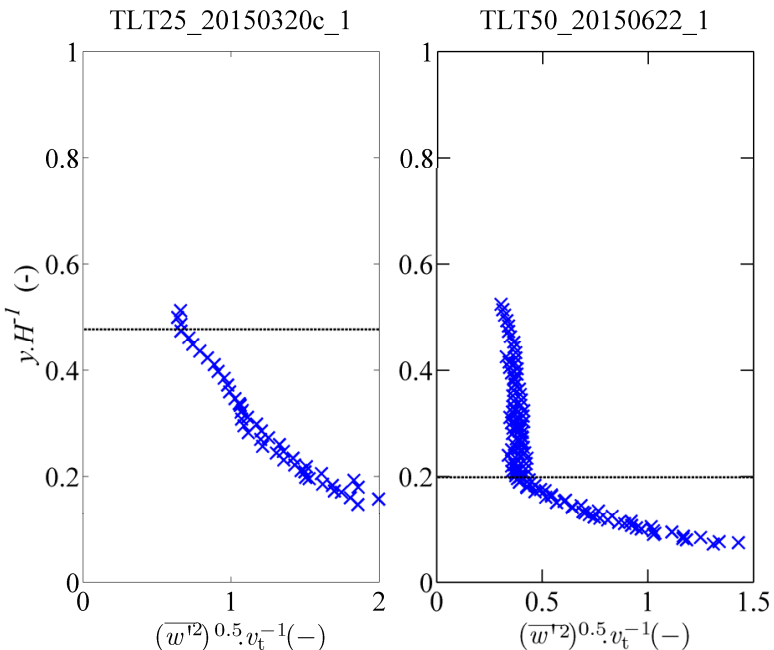

Figure 6. Distribution of vertical turbulent intensity (fluctuation normalized by local hindered settling velocity). Legend: $\mathrm{x}-$ ADVP, line - visually observed top of transport layer.

\subsubsection{Reynolds stress}

The local Reynolds stress could be evaluated from the ADVP measurements only just as the vertical turbulence intensity. Plotted in Figure 7, the Reynolds stress obtained from measured velocity components using Eq. (4) is normalized by the square of $u_{* \text { int }}$ and the density of water. In Figure 7, the inclined line delineates the theoretical distribution of total applied shear stress in uniform flow above the bed [9]. The linear course and the maximum value of 1 at the flow boundary follows from the integral force balance on the control volume of flow. In the right-side panels of Figure 7, experimentally determined Reynold stresses exhibit a linear distribution at vertical positions above the transport layer and a change in the trend at the top of the transport layer. In the transport layer, the Reynolds stress decreases towards a zero value at the top of the bed. The linear trend of the data above the transport layer indicates that the Reynolds stress is the exclusive local resisting stress. This is not the case within the transport layer, where transported particles through their mutual collisions take care of a part of the local stress. Frequency of collisions increases with increasing local concentration of particles and so increases the local particulate stress. Hence the momentum transfer due to collisions takes a more important role in the total shear stress transfer. This is indicated by the increasing difference between the measured Reynolds stress and the total shear stress with the increasing depth in the transport layer. This decrease in the Reynolds stress can be interpreted as a result of damping of turbulence in the layer due to particles. The left-side panels of Figure 7 confirm the observed trends for flow of the smaller depth and higher bed shear stress. A value of $u_{* \text { opt }}$ is indicated by the red dot in Figure 7. The horizontal ordinate of the dot gives $\left(u *_{\text {opt }} / u_{* \text { int }}\right)^{2}$. The vertical ordinate of the dot indicates the virtual origin of the log-profile, $y_{\text {ini }} / H$.
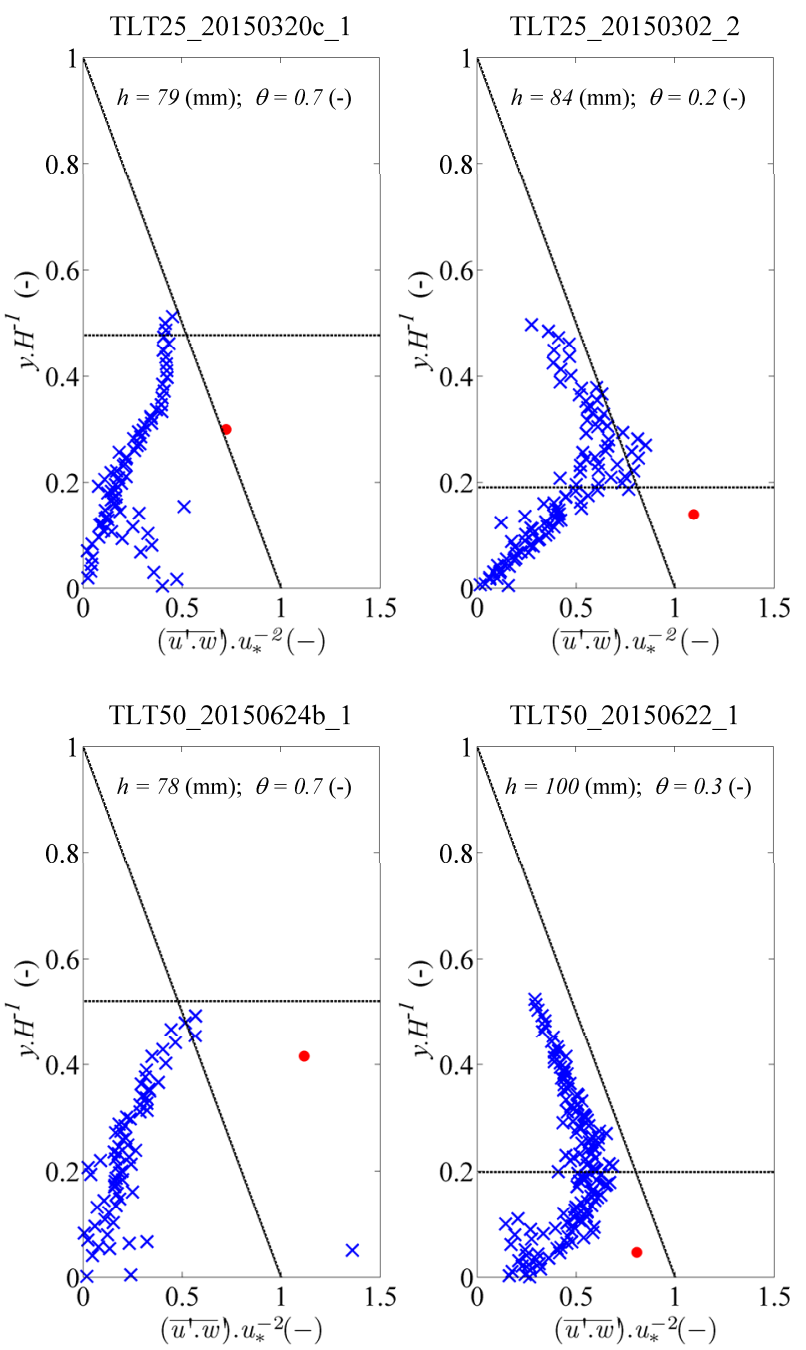

Figure 7. Distribution of shear stresses (stress normalised by square of shear velocity evaluated from integral data). Legend: crosses - Reynolds stress measured by ADVP, dot - value obtained by optimization of parameters of theoretical velocity profile, inclined line - theoretical distribution of total shear stress, horizontal line - visually observed position of top of transport layer.

\subsubsection{Particulate stress in transport layer}

As discussed above, the difference between the local Reynolds stress and the local total shear stress is the local particulate shear stress transferred by interparticle collisions. This collisional stress is plotted in Figure 8 (green dots) and it seems to exhibit an approximately linear distribution across the transport layer (see the fit by 
a red line). The position of the zero collisional stress (i.e. the position of the intersection of the red line with the $y$ axis) marks a position above which no particles are supported by mutual contacts. Since there are no particles supported by turbulent eddies at this position either (Fig. 6 ), no particles are expected to be present above the position of the zero collisional stress and hence this position should be the vertical position of the top of the transport layer. This is a method of a determination of the top of the transport layer alternative to the method of the visual determination of the top of the layer.

The Reynolds stress decreases towards zero at the bottom of the transport layer indicating that the applied shear stress is resisted exclusively by the particulate stress at the top of the bed. Also at the top of the bed, the collisional particulate shear stress $\tau_{0}$ is related to the interparticle normal stress $\sigma_{0}$ through the Coulomb criterion (e.g. Capart and Fraccarollo [6])

$$
\tau_{0}=\tan (\alpha) \cdot \sigma_{0}
$$

where $\alpha$ is the quasi-static internal angle of friction (Bagnold [12] reported values of $\tan (\alpha)$ to vary from 0.32 to 0.75 in his experiments) and the normal stress is expressed using parameters associated with the transport layer,

$$
\sigma_{0}=\left(\rho_{s}-\rho_{w}\right) \cdot H_{s h} \cdot g \cdot C_{T L} \cdot \cos (\beta)
$$

in which $C_{\mathrm{TL}}$ is mean volumetric concentration of particles in the transport layer (typically one half of local concentration at the bottom of the transport layer) and $H_{\mathrm{sh}}$ is thickness of transport layer. A combination of Eqs. (8a) and (8b) gives a formula for $\tan (\alpha)$,

$$
\tan (\alpha)=\frac{\tau_{0}}{H_{s h} \cdot\left(\rho_{s}-\rho_{w}\right) \cdot g \cdot C_{T L} \cdot \cos (\beta)}
$$

Based on results of our experiments and estimated $C_{\mathrm{TL}}=$ 0.27 , we obtained values of $\tan (\alpha)$ in the range from 0.2 to 0.44 from Eq. (9).

\section{Conclusions}

A large experimental data set of vertical velocity profiles was analysed for layered flows with both relatively weak and intense transport of sediment. The data confirm that a velocity profile can be considered composed of a linear part and a logarithmic part in flow with a developed transport layer.

Turbulent characteristics evaluated from acoustic Doppler-based measurements indicated that turbulent eddies might not be able to support sediment particles at the top of the transport layer, i.e. the sediment was transported predominantly as bed load. Furthermore, an analysis of turbulent velocity fluctuations in the transport layer suggested that an interaction of turbulent eddies with colliding particles leads to attenuation of both the longitudinal turbulent intensity and the Reynolds stress. The measure of attenuation increases with the increasing local concentration of particles. At the bottom of the transport layer, the Reynolds stress is negligible and the applied shear stress is resisted exclusively by the particulate shear stress.

The angle of internal friction of colliding particles was evaluated to vary from 0.22 to 0.44 at the bottom of the transport layer.
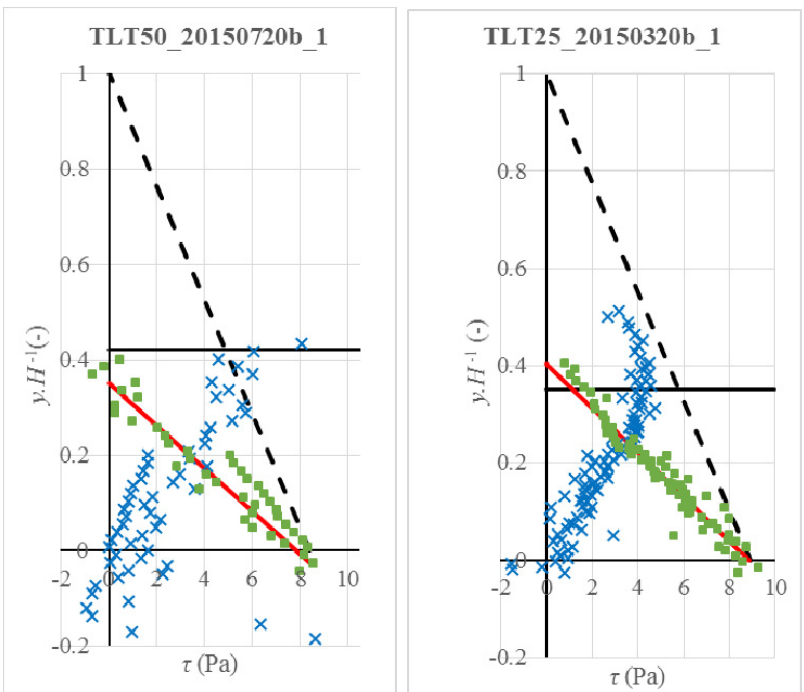

Figure 8. Distribution of shear stresses. Legend: crosses Reynolds stress, squares - calculated collisional shear stress, dashed line - theoretical distribution of total shear stress, red line - linear fit of collisional shear stress, horizontal line - the visually observed interface between water and transport layer.

\section{Acknowledgments}

The research has been supported by the Czech Science Foundation through the grant project No. P105/12/1082 and by Faculty of Civil Engineering of the Czech Technical University in Prague through the student grant project No. SGS14/179/OHK1/3T/11.

\section{References}

1. D. Rickenman, A. Koschni, Hydrol. Process., 24, 931007, (2010)

2. V. Matoušek, J. Krupička, Proc. River Flow 2014, Lausanne, Switzerland, p. 917-922, (2014).

3. V. Matoušek, V. Bareš. J. Krupička, T. Picek, Š. Zrostlík, J. Hydrol. Hydromech., 63(4), in press, (2015)

4. Š. Zrostlík, V. Bareš, J. Krupička, T. Picek, V. Matoušek, EPJ Web of Conferences 92, 02120 (2015)

5. V. Matoušek, J. Hydraul. Eng.-ASCE, 135(12), 1101-1106, (2009)

6. H. Capart, L. Fraccarollo, Geophysi. Res. Lett., 38, (2011)

7. A. Yeganeh, H. Gotoh, T. Sakai, J. Hydraul. Res., 38(5), 389-398, (2000)

8. J. K. Rajesh, A. Kumar, U. Kothyari, Journal of Hydro-environment Research, in press, (2015) 
9. T. Song, H. W. Graf, U. Lemin, J. Hydraul. Res., 32(6), 861-876, (1994)

10. T. Revil-Baudard, J. Chauchat, D. Hurther, P-A.

Barraud, J. Fluid Mech., 767, 1-30, (2015)

11. P. Bakhtyar, A. Yeganeh-Bakhtiary, D.A. Barry, A. Ghaheri, Advances in Water Resources, 32, 12671283, (2009)

12. R. A. Bagnold, U.S. Geological Survey Professional Paper, 422-I, p. 37, (1966) 